\title{
Tradução, adaptação, validade e medidas de fidedignidade do Instrumento de Avaliação da Atenção Primária à Saúde (PCATool) no Brasil: versão profissionais de saúde
}

\author{
Translation, adaptation, validity and reliability of the Instrument for Assessment of Primary Health Care \\ (PCATool) in Brazil: version of health professionals
}

\section{Traducción, adaptación, validez y confiabilidad del instrumento de evaluación de la Atención Primaria de Salud (PCATool) en Brasil: versión profesionales de la salud}

\begin{abstract}
Lisiane Hauser. Universidade Federal do Rio Grande do Sul (UFRGS). Porto Alegre, RS, Brasil. lisiane.hauser@ymail.com (Autora correspondente) Rodrigo Caprio Leite de Castro. Universidade Federal do Rio Grande do Sul (UFRGS). Porto Alegre, RS, Brasil. rodrigo_caprio@yahoo.com.br Álvaro Vigo. Universidade Federal do Rio Grande do Sul (UFRGS). Porto Alegre, RS, Brasil. alvaro.vigo@gmail.com

Thiago Gomes da Trindade. Universidade Federal do Rio Grande do Norte (UFRN). Natal, RN, Brasil. thiagogtrindade@gmail.com Marcelo Rodrigues Gonçalves. Universidade Federal do Rio Grande do Sul (UFRGS). Porto Alegre, RS, Brasil. marcelorog@gmail.com Airton Tetelbom Stein. Universidade Federal de Ciências da Saúde de Porto Alegre (UFCSPA). Porto Alegre, RS, Brasil. airton.stein@gmail.com Bruce Bartholow Duncan. Universidade Federal do Rio Grande do Sul (UFRGS). Porto Alegre, RS, Brasil. bbduncan@ufrgs.br Erno Harzheim. Universidade Federal do Rio Grande do Sul (UFRGS). Porto Alegre, RS, Brasil. ernoharz@terra.com.br
\end{abstract}

\section{Resumo}

Objetivo: traduzir, adaptar e avaliar a validade e a fidedignidade do PCATool versão profissionais de saúde utilizando um estudo transversal com médicos e enfermeiros $(n=340)$ dos serviços públicos de APS em Porto Alegre. Métodos: foram realizadas tradução, versão e adaptação do instrumento às características dos serviços de saúde nacionais, bem como culturais. A validade fatorial, a consistência interna e a estabilidade no tempo foram avaliadas. Resultados: na análise fatorial exploratória foram retidos 9 fatores, explicando aproximadamente $75 \%$ da variação total. Dessa forma, foi identificada a estrutura multidimensional do instrumento. A avaliação da consistência interna mostrou coeficiente alfa de Cronbach variando de 0,28 a 0,90. A estabilidade temporal foi observada para todos os atributos à exceção de Orientação Familiar $(p<0,05)$. Conclusões: essa avaliação sugere que o instrumento é válido e fidedigno para a mensuração da qualidade dos serviços de atenção primária à saúde, na perspectiva dos profissionais de saúde. Pode ser utilizado tanto para identificação, monitoramento e avaliação dos atributos da APS nos serviços de saúde quanto para estudos comparativos.

\section{Abstract}

Objective: to translate and adapt the version of the Primary Care Assessment Tool (PCATool) for health professionals in the Brazilian context and assess its validity and reliability. Methods: we conducted a cross-sectional study with 340 healthcare professionals (physicians and nurses) working in the public primary care services in Porto Alegre. We performed the translation, reverse translation, and adaptation of the instrument to the characteristics of the national health service, as well as to cultural aspects. Factorial validity, internal consistency, and stability over time were assessed. Results: nine factors were retained in the exploratory factorial analysis, explaining approximately $75 \%$ of the total variation, highlighting the multi-dimensional nature of the instrument. In terms of internal consistency, Cronbach's alpha ranged from 0.28 to 0.90 . Temporal stability was observed for all attributes except family counseling $(p<0.05)$. Conclusions: the results suggest that the PCATool can be useful in measuring the quality of primary care from the perspective of healthcare professionals, being applicable not only for identification, assessment, and screening of primary care attributes within healthcare services, but also for comparative studies.

Palavras-chave: Atenção Primária à Saúde Avaliação de Serviços de Saúde Validade dos Testes Reprodutibilidade dos Testes

\section{Keywords:}

Primary Health Care Health Services Evaluation Validity of Tests Reproducibility of Results 


\section{Resumen}

Objetivos: traducir, adaptar y evaluar la validez y fiabilidad del PCATool versión profesionales de la salud mediante un estudio transversal con médicos y enfermeros ( $n=340)$ de los servicios públicos de APS en Porto Alegre. Métodos: se realizó la traducción, versión y adaptación del instrumento a las características de los servicios nacionales de salud, así como culturales. Se evaluaron la validez factorial, la consistencia interna y la estabilidad en el tiempo. Resultados: en el análisis factorial exploratorio se retuvieron nueve factores, lo que representa aproximadamente el 75\% de la variación total. De esa forma, se identificó la estructura multidimensional del instrumento. La evaluación de la consistencia interna mostró el coeficiente alfa de Cronbach variando entre 0,28 y 0,90. Se observó estabilidad temporal en todos los atributos excepto en la Orientación Familiar ( $p<0,05)$. Conclusiones: esta evaluación sugiere que el instrumento es válido y fiable para medir la calidad de los servicios de atención primaria de la salud, desde la perspectiva de los profesionales de la salud. Puede ser utilizado tanto para la identificación, seguimiento y evaluación de los atributos de APS en los servicios de salud como para los estudios comparativos.
Palabras clave:

Atención Primaria de Salud Evaluación de Servicios de Salud Validez de las Pruebas Reproducibilidad de Resultados

\section{Introdução}

A Atenção Primária à Saúde (APS) se consolidou como uma das formas mais equitativas e eficientes de organizar um sistema de saúde ${ }^{1}$. A partir da década de 90 , foi observado um aumento substancial de estudos evidenciando o impacto positivo de sistemas de saúde orientados pela APS na provisão de melhores cuidados em saúde para suas populaçóes ${ }^{2}$. Neste período, foi instituído no Brasil o Programa Saúde da Família que, posteriormente, teve seu conceito ampliado e foi denominado Estratégia Saúde da Família (ESF). Essa estratégia foi adotada pelo Ministério da Saúde para a reorientação e expansão da APS no Brasil e, consequentemente, reorganizaçáo do Sistema Único de Saúde (SUS). Assim, diante do notável avanço da APS, torna-se relevante avaliar os resultados alcançados em relação à organização e provisão dos serviços, e também no que se refere aos possíveis impactos produzidos na saúde e bem-estar da população.

Diferentes modelos conceituais foram desenvolvidos para avaliar a qualidade da atenção à saúde, alguns também incluindo os atributos da APS ${ }^{3-5}$. A partir disso, o crescente interesse na avaliaçáo e melhoria da gestáo, do planejamento e da qualidade na atenção à saúde tem estimulado o desenvolvimento de um grande número de instrumentos para avaliar os serviços de saúde na perspectiva dos usuários, profissionais de saúde e gestores ${ }^{6}$. Alguns desses instrumentos são especialmente voltados para a avaliação da qualidade da $\mathrm{APS}^{7}$, porém poucos permitem a ampla avaliação dos atributos que definem a APS na perspectiva da população (Acesso de Primeiro Contato, Longitudinalidade, Integralidade e Coordenação) ${ }^{8-10}$.

Nesse contexto, destaca-se o instrumento Primary Care Assessment Tool (PCATool), desenvolvido para medir o grau de orientação à APS considerando o quadro conceitual baseado nos atributos essenciais - Acesso de Primeiro Contato, Longitudinalidade, Integralidade e Coordenação -; e derivados - Orientação Familiar, Orientação Comunitária e Competência Cultural ${ }^{11,12}$. Esse instrumento pode ser respondido por um responsável pelas crianças usuárias de um serviço (Child Consumer), por usuários adultos (Adult Consumer), por profissionais de saúde (Provider Survey) e por gestores (Facility Survey). As versões originais para usuários crianças e usuários adultos foram validadas nos Estados Unidos ${ }^{9,11}$. Para profissionais de saúde, é usual a utilização de uma versão como espelho da versão para usuários adultos validada ${ }^{12}$, isto é, os itens que estão presentes na versão usuários adultos validada, acrescida de alguns itens da versão para usuários crianças, devem estar presentes na versão profissionais de saúde.

O PCATool foi traduzido e adaptado em vários países, com diferentes sistemas de saúde, incluindo o Brasil ${ }^{13-15}$, a Espanha ${ }^{16}$, o Canadá $^{17}$, a Coreia do Sul ${ }^{18}$, Hong Kong ${ }^{19}$ e a Argentina ${ }^{20}$. Está em fase de tradução e adaptação em Porto Rico ${ }^{21}$ e no Uruguai $^{22}$ e vem sendo utilizado na Nova Zelândia ${ }^{23}$ e em Taiwan ${ }^{24}$. As versões utilizadas na Espanha ${ }^{16,25}$, na Coreia do Sul ${ }^{18}$ e no Brasil ${ }^{13-15}$ foram validadas com algumas variaçóes na metodologia adotada. Os resultados foram adequados, respeitando a estrutura multidimensional na versão original e a unidimensionalidade para a redução do instrumento.

No Brasil, particularmente, foram realizadas duas adaptaçôes distintas deste instrumento, bem como processos diferentes de avaliação da validade e fidedignidade. A adaptação realizada por Harzheim et al. ${ }^{13,14}$ foi aplicada em Porto Alegre, Rio Grande do Sul, mantendo o formato original do instrumento, e avaliou a validade e a fidedignidade das versóes usuários crianças $^{13}$ e usuários adultos ${ }^{14}$. Por outro lado, uma adaptação realizada por Almeida e Macinko ${ }^{15}$ foi utilizada em Petrópolis, Rio de Janeiro, e resultou na validação das versôes usuários crianças, usuários adultos e profissionais de saúde.

A mensuração da qualidade dos serviços de saúde deve ser realizada com instrumentos válidos e fidedignos, uma vez que a informação sobre as propriedades psicométricas permite conhecer a acurácia e a precisão da medida da qualidade de atenção ao cuidado. Além disso, a implantação da ESF não assegura a efetiva aplicação dos princípios da APS nos serviços de saúde, tornando relevante a avaliação da presença e extensão dos atributos da APS, que são internacionalmente reconhecidos 
como eixos estruturantes do processo de atenção associados à qualidade dos serviços, à efetividade e à eficiência de suas intervençôes ${ }^{26}$.

O objetivo deste estudo foi traduzir, adaptar e avaliar a validade e a fidedignidade do instrumento de avaliaçáo da qualidade na APS, versão profissionais de saúde.

\section{Métodos}

\section{Delineamento e amostra}

Foi realizado um estudo transversal de base populacional com profissionais médicos e enfermeiros dos serviços públicos de APS no município de Porto Alegre. No período da coleta de dados (de julho de 2006 a agosto de 2007), a rede pública de APS neste município era constituída por quatro serviços de saúde: Unidades Básicas de Saúde tradicionais (UBS), Unidades da Estratégia Saúde da Família (ESF), Unidades do Centro de Saúde Escola Murialdo (CSEM) e Unidades do Serviço de Saúde Comunitária (SSC) do Grupo Hospitalar Conceição (GHC).

Foram incluídos na amostra todos os médicos e enfermeiros que atendiam, pelo menos, usuários adultos (maiores de 18 anos) e informaram carga horária mínima de 20 horas semanais. Os profissionais que realizavam atendimento somente a partir de encaminhamentos (referências) foram excluídos. Detalhes do plano amostral e seleção dos profissionais são encontrados no protocolo de pesquisa ${ }^{27}$.

\section{Instrumento de Avaliação da Atenção Primária à Saúde (PCATool)}

O instrumento original do PCATool para profissionais de saúde está disponível em inglês e é constituído por 124 itens ${ }^{4}$, sendo 5 itens específicos sobre o cuidado com as crianças, os quais são respondidos somente pelos profissionais que atendem crianças. Os itens desse instrumento estão distribuídos nos quatro atributos essenciais da APS, permitindo avaliar o Acesso de Primeiro Contato, a Longitudinalidade (a relação profissional com o paciente ao longo do tempo), a Coordenação dos serviços e a Integralidade dos Serviços Disponíveis e Prestados. Os três atributos derivados da APS também são contemplados, possibilitando a avaliação da Orientação Familiar, Orientação Comunitária e Competência Cultural. Cada atributo essencial é constituído por dois componentes, um relacionado à estrutura e o outro ao processo de cuidado ${ }^{12}$.

A escala de respostas dos itens é do tipo Likert ( $4=$ com certeza sim, $3=$ provavelmente sim, $2=$ provavelmente não, $1=$ com certeza não), com o acréscimo da opção ' $9=$ não sei/não lembro' (nas análises deste artigo o valor 9 foi recodificado para o valor 2,5) ${ }^{12}$. A partir dessas respostas, é possível calcular um escore para cada atributo da APS (e de seus componentes) e também um Escore Essencial e um Escore Geral. Os escores para cada atributo (bem como de seus componentes) são obtidos pela média aritmética das respostas dos seus respectivos itens. O Escore Essencial consiste na média aritmética dos escores dos atributos essenciais e o Escore Geral é definido pela média aritmética dos escores dos atributos essenciais e derivados.

\section{Tradução e adaptação}

O PCATool foi traduzido para o português e, posteriormente, foi realizada a versão para o inglês. Os dois documentos em inglês foram então comparados para corrigir falhas na tradução para o português. A tradução e a versão para o inglês foram realizadas por uma profissional nativa da língua inglesa, com domínio da língua portuguesa. Originalmente desenvolvido para ser autoaplicado, o instrumento traduzido para o português foi adaptado para ser aplicado por entrevistadores e também para as características culturais e dos serviços de saúde nacionais. Na etapa seguinte, o instrumento foi aplicado a seis profissionais de saúde (5 médicos e 1 enfermeira) com o objetivo de avaliar a adaptaçáo da forma e do vocabulário.

A validade de conteúdo, isto é, a adequação com a qual uma medida afere o atributo de interesse, foi realizada por dois pesquisadores e pela autora original do instrumento, constituindo a avaliação conceitual dos atributos. 


\section{Análise estatística}

A análise fatorial exploratória foi realizada para observar se os itens que constituem um atributo teórico apresentam convergência empírica, representando o constructo teórico que se quer medir (validade fatorial). A extração dos fatores foi efetuada via componentes principais, utilizando o maior coeficiente de correlação de Pearson entre os itens como estimativas iniciais das comunalidades. O método ortogonal Equamax foi utilizado para a rotação dos fatores.

A fidedignidade das escalas foi observada pela consistência interna, razão do êxito da escala (REE) e estabilidade no tempo. Para as análises relacionadas à fidedignidade, foi considerada a distribuição dos itens em cada atributo, respeitando a conceituação teórica proposta por Starfield .

A consistência interna foi verificada utilizando o coeficiente alfa de Cronbach e a correlação item-total. Para a interpretação do alfa de Cronbach, valores inferiores a 0,50 foram considerados insuficientes, valores entre 0,50 e 0,70 moderados e valores acima de 0,70 adequados. Na correlação item-total, os valores maiores que 0,30 sugerem que os itens estão medindo o mesmo constructo e são, portanto, considerados adequados ${ }^{28}$.

$\mathrm{Na}$ REE a correlação de cada item com os outros itens no seu atributo conceitual deve ser maior que a correlação com itens de diferentes atributos. Assim, a REE foi medida pelo quociente entre o número de vezes que correlaçóes entre os itens de um atributo foram superiores às correlaçóes de cada um desses itens com os outros atributos (numerador) e o total de correlaçóes efetuadas nesse atributo. Isto é, o denominador é definido pelo produto entre o número de itens do atributo e o número de atributos. O resultado é apresentado em percentual e valores elevados dessa medida sugerem maior discriminação do atributo ${ }^{9}$.

$\mathrm{Na}$ avaliação da fidedignidade (estabilidade no tempo), utilizaram-se aproximadamente $10 \%$ das entrevistas que foram repetidas entre um e seis meses após a sua primeira realização. O teste Wilcoxon para amostras pareadas e o procedimento de Bland-Altman, baseado no teste t para amostras pareadas, foram utilizados nessas análises.

Os itens que constituem o atributo Competência Cultural não foram considerados para as análises, uma vez que esse atributo não foi validado no Brasil na versão usuário criança ${ }^{13}$, nem na versão usuário adulto ${ }^{14}$. O programa $\mathrm{R}$ foi utilizado para as análises de estabilidade no tempo (Bland-Altman). As demais análises foram realizadas com o programa SAS (Statistical Analysis Software) versão 9.2. O nível de significância 5\% foi considerado em todas as análises.

\section{Aspectos éticos}

O projeto principal intitulado "Avaliação da qualidade do processo de atenção e da sua efetividade sobre a saúde do adulto no Programa Saúde da Família e modelos alternativos no município de Porto Alegre" teve aprovaçáo dos comitês de ética das instituiçóes envolvidas na pesquisa: Secretaria Municipal de Saúde (SMS), Centro de Saúde Escola Murialdo (CSEM), Grupo Hospitalar Conceição (GHC), Grupo de Pesquisa e Pós Graduação - Hospital de Clínicas de Porto Alegre (GPPGHCPA), Caixa de Assistência dos Funcionários do Banco do Brasil - Regional RS (CASSI-RS) e Universidade Federal do Rio Grande do Sul-UFRGS. As entrevistas foram realizadas com os adultos mediante a leitura, aceitação e assinatura do Termo de Consentimento Livre e Esclarecido (TCLE).

\section{Resultados}

Na comparação da versão do PCATool traduzida para o português com a versão original, não foram observadas diferenças de conteúdo ou acepção. A escala de respostas foi fielmente traduzida para o português. No processo de adaptação, 3 itens de Integralidade de Serviços Disponíveis (testes de intoxicação por chumbo, timpanocentese e exame de toque retal ou sigmoidoscopia) e 2 itens de Competência Cultural (possibilidade de comunicação com pessoas em outro idioma e uso de tradutores/intérpretes) foram excluídos, porque estavam relacionados a açôes não disponíveis nas unidades de saúde brasileiras. Assim, ao final da etapa de tradução e adaptação, o PCATool-Brasil para profissionais de saúde foi composto por 119 itens distribuídos nos quatro atributos essenciais e três atributos derivados, da mesma maneira que o instrumento original ${ }^{12}$. 
Foram selecionados 369 profissionais médicos e enfermeiros. Houve recusa por 20 profissionais e, para outros 9, a entrevista não foi realizada por troca de unidade, afastamento por motivo de saúde ou demissão, totalizando perda de $29(7,9 \%)$ entrevistas. Dessa forma, a amostra em estudo foi de 340 profissionais nos quatro serviços de saúde (n): UBS (161), ESF (85), SSC do GHC (72) e CSEM (22). Foram entrevistados 226 (66,5\%) médicos e 114 (33,5\%) enfermeiros. A idade média (desvio padrão) desses profissionais foi de 43,6 anos (9,4 anos), sendo 97 (28,5\%) homens. O tempo médio (desvio padrão) de formado foi 17,1 anos (9,3 anos). A maioria dos profissionais, 297 (87,4\%), possuía alguma especialidade e, desses, $113(33,2 \%)$ referiram ter especialidade na área da APS.

$\mathrm{Na}$ análise fatorial exploratória, foram retidos 9 fatores, explicando aproximadamente $75 \%$ da variação total. Esses fatores representaram de forma mais homogênea os atributos que possibilitam mensurar a presença e extensão da APS. As cargas fatoriais são apresentadas na última coluna da Tabela 1. Observou-se que todos os itens de Orientação Familiar foram captados em um único fator, da mesma forma que aconteceu com a Integralidade de Serviços Prestados. A Orientação Comunitária foi captada em dois fatores, assim como a Integralidade de Serviços Prestados. O Acesso de Primeiro Contato apresentou 4 itens em um fator e os outros 5 itens do Acesso de Primeiro Contato foram captados no mesmo fator em que estão 6 itens da Longitudinalidade. Os demais itens da Longitudinalidade formaram outro fator em conjunto com itens da Coordenação (Tabela 2).

Tabela 1. Distribuição das respostas dos itens, escore médio e cargas fatoriais para validade fatorial.

\begin{tabular}{|c|c|c|c|c|c|c|c|}
\hline Itens por atributos & $\begin{array}{l}\text { Não sei/Não } \\
\text { lembro n (\%) }\end{array}$ & $\begin{array}{l}\text { Com certeza } \\
\text { não n (\%) }\end{array}$ & $\begin{array}{c}\text { Provavelmente } \\
\text { não n (\%) }\end{array}$ & $\begin{array}{l}\text { Provavelmente } \\
\operatorname{sim} \mathrm{n}(\%)\end{array}$ & $\begin{array}{l}\text { Com certeza } \\
\operatorname{sim} n(\%)\end{array}$ & $\begin{array}{l}\text { Escore } \\
\text { Médio\# }\end{array}$ & $\begin{array}{r}\text { Carga } \\
\text { fatorial }\end{array}$ \\
\hline \multicolumn{8}{|l|}{ Acesso - Primeiro Contato } \\
\hline Aberto sábado ou domingo & $1(0,3)$ & $331(97,4)$ & $4(1,2)$ & $4(1,2)$ & $0(0,0)$ & 1,04 & 0,12 \\
\hline $\begin{array}{l}\text { Aberto, pelo menos em alguns dias da semana até } \\
\text { as } 20 \mathrm{hs}\end{array}$ & $4(1,2)$ & $303(89,1)$ & $2(0,6)$ & $7(2,1)$ & $24(7,1)$ & 1,28 & $-0,01$ \\
\hline $\begin{array}{l}\text { Quando aberto, alguém do seu serviço } 0 \text { atenderia no } \\
\text { mesmo dia }\end{array}$ & $3(0,9)$ & $2(0,6)$ & $11(3,2)$ & $121(35,6)$ & $203(59,7)$ & 3,55 & 0,33 \\
\hline $\begin{array}{l}\text { Quando aberto, aconselhamento rápido pelo telefone } \\
\text { se necessário }\end{array}$ & $24(7,1)$ & $26(7,7)$ & $61(17,9)$ & $132(38,9)$ & $97(28,5)$ & 2,92 & 0,43 \\
\hline $\begin{array}{l}\text { Quando fechado existe um número de telefone para } \\
\text { contato }\end{array}$ & $17(5,0)$ & $278(81,8)$ & $20(5,9)$ & $9(2,7)$ & $16(4,7)$ & 1,33 & 0,11 \\
\hline $\begin{array}{l}\text { Quando fechado sábados e domingos, atendimento } \\
\text { naquele dia por alguém do serviço }\end{array}$ & $1(0,3)$ & $309(90,9)$ & $24(7,1)$ & $5(1,5)$ & $1(0,3)$ & 1,11 & 0,59 \\
\hline $\begin{array}{l}\text { Quando fechado à noite, atendimento naquela noite } \\
\text { por alguém do serviço }\end{array}$ & $1(0,3)$ & $309(90,9)$ & $22(6,5)$ & $7(2,1)$ & $1(0,3)$ & 1,12 & 0,56 \\
\hline É fácil marcar consulta de revisão & $6(1,8)$ & $34(10,0)$ & $54(15,9)$ & $144(42,4)$ & $102(30,0)$ & 2,93 & 0,26 \\
\hline $\begin{array}{l}\text { Esperar mais de } 30 \text { minutos para serem atendidos } \\
\text { pelo médico ou pela enfermeira }\end{array}$ & $5(1,5)$ & $81(23,8)$ & $110(32,4)$ & $100(29,4)$ & $44(12,9)$ & 2,68 & 0,02 \\
\hline \multicolumn{8}{|l|}{ Longitudinalidade } \\
\hline Atendimento pelo mesmo médico/enfermeira & $5(1,5)$ & $71(20,9)$ & $64(18,8)$ & $135(39,7)$ & $65(19,1)$ & 2,58 & 0,16 \\
\hline $\begin{array}{l}\text { Entendimento das perguntas que seus pacientes the } \\
\text { fazem }\end{array}$ & $0(0,0)$ & $0(0,0)$ & $0(0,0)$ & $82(24,1)$ & $258(75,9)$ & 3,76 & 0,41 \\
\hline $\begin{array}{l}\text { Entendimento dos pacientes do quê você diz ou } \\
\text { pergunta a eles }\end{array}$ & $2(0,6)$ & $0(0,0)$ & $3(0,9)$ & $213(62,7)$ & $122(35,9)$ & 3,35 & 0,45 \\
\hline $\begin{array}{l}\text { Pacientes podem telefonar e falar com médico ou } \\
\text { enfermeira que os conhece melhor }\end{array}$ & $6(1,8)$ & $40(11,8)$ & $79(23,2)$ & $107(31,5)$ & $108(31,8)$ & 2,84 & 0,38 \\
\hline $\begin{array}{l}\text { Tempo suficiente aos pacientes para discutirem seus } \\
\text { problemas ou preocupações }\end{array}$ & $2(0,6)$ & $10(3,0)$ & $29(8,5)$ & $144(42,4)$ & $155(45,6)$ & 3,31 & 0,35 \\
\hline $\begin{array}{l}\text { Pacientes se sentem confortáveis ao lhe contar suas } \\
\text { preocupações ou problemas }\end{array}$ & $3(0,9)$ & $0(0,0)$ & $1(0,3)$ & $154(45,3)$ & $182(53,5)$ & 3,53 & 0,36 \\
\hline $\begin{array}{l}\text { Conhece "muito bem" os pacientes de seu serviço de } \\
\text { saúde }\end{array}$ & $3(0,9)$ & $20(5,9)$ & $71(20,9)$ & $179(52,7)$ & $67(19,7)$ & 2,87 & 0,46 \\
\hline Sabe quem mora com cada um de seus pacientes & $5(1,5)$ & $102(30,0)$ & $96(28,2)$ & $100(29,4)$ & $37(10,9)$ & 2,22 & 0,43 \\
\hline
\end{tabular}


Tabela 1. Distribuição das respostas dos itens, escore médio e cargas fatoriais para validade fatorial.

\begin{tabular}{|c|c|c|c|c|c|c|c|}
\hline Itens por atributos & $\begin{array}{l}\text { Não sei/Não } \\
\text { lembro n (\%) }\end{array}$ & $\begin{array}{c}\text { Com certeza } \\
\text { não } \mathrm{n}(\%)\end{array}$ & $\begin{array}{l}\text { Provavelmente } \\
\text { não } \mathrm{n}(\%)\end{array}$ & $\begin{array}{l}\text { Provavelmente } \\
\operatorname{sim} \mathrm{n}(\%)\end{array}$ & $\begin{array}{c}\text { Com certeza } \\
\operatorname{sim} n(\%)\end{array}$ & $\begin{array}{l}\text { Escore } \\
\text { Médio" }\end{array}$ & $\begin{array}{c}\text { Carga } \\
\text { fatorial }^{*}\end{array}$ \\
\hline $\begin{array}{l}\text { Entende quais problemas são os mais importantes } \\
\text { para os pacientes }\end{array}$ & $4(1,2)$ & $5(1,5)$ & $14(4,1)$ & $227(66,8)$ & $90(26,5)$ & 3,19 & 0,25 \\
\hline $\begin{array}{l}\text { Conhece o histórico médico completo de cada } \\
\text { paciente }\end{array}$ & $3(0,9)$ & $51(15,0)$ & $99(29,1)$ & $155(45,6)$ & $32(9,4)$ & 2,50 & 0,66 \\
\hline Sabe qual o trabalho ou emprego de cada paciente & $2(0,6)$ & $84(24,8)$ & $113(33,3)$ & $111(32,7)$ & $29(8,6)$ & 2,25 & 0,58 \\
\hline $\begin{array}{l}\text { Saberia sobre pacientes com dificuldades para obter } \\
\text { ou pagar por medicamentos }\end{array}$ & $1(0,3)$ & $13(3,82)$ & $27(7,94)$ & $168(49,4)$ & $131(38,5)$ & 3,23 & 0,28 \\
\hline $\begin{array}{l}\text { Sabe todos os medicamentos que pacientes estão } \\
\text { tomando }\end{array}$ & $1(0,3)$ & $39(11,5)$ & $50(14,7)$ & $141(41,5)$ & $109(32,1)$ & 2,94 & 0,60 \\
\hline \multicolumn{8}{|l|}{ Coordenação do Cuidado } \\
\hline $\begin{array}{l}0 \text { serviço comunica ou entrega todos os resultados } \\
\text { dos exames laboratoriais }\end{array}$ & $8(2,4)$ & $45(13,2)$ & $22(6,5)$ & $85(25,0)$ & $180(52,9)$ & 3,19 & 0,12 \\
\hline Sabe sobre consultas de pacientes com especialistas & $7(2,1)$ & $60(17,6)$ & $89(26,2)$ & $118(34,7)$ & $66(19,4)$ & 2,57 & 0,49 \\
\hline $\begin{array}{l}\text { Discute com os pacientes sobre diferentes serviços } \\
\text { especializados }\end{array}$ & $1(0,3)$ & $52(15,3)$ & $48(14,1)$ & $89(26,2)$ & $150(44,1)$ & 2,99 & 0,28 \\
\hline $\begin{array}{l}\text { Auxílio do serviço para marcar a consulta } \\
\text { encaminhada (ao especialista) }\end{array}$ & $5(1,5)$ & $8(2,4)$ & $3(0,9)$ & $57(16,8)$ & $267(78,5)$ & 3,72 & 0,07 \\
\hline Informação escrita para levar ao especialista & $0(0,0)$ & $2(0,6)$ & $1(0,3)$ & $29(8,5)$ & $308(90,6)$ & 3,89 & 0,28 \\
\hline $\begin{array}{l}\text { Recebe do especialista informações úteis sobre } 0 \\
\text { paciente encaminhado }\end{array}$ & $0(0,0)$ & $78(22,9)$ & $166(48,8)$ & $73(21,5)$ & $23(6,8)$ & 2,12 & 0,18 \\
\hline $\begin{array}{l}\text { Após a consulta com o especialista, conversa sobre os } \\
\text { resultados desta consulta }\end{array}$ & $0(0,0)$ & $7(2,1)$ & $20(5,9)$ & $125(36,8)$ & $188(55,3)$ & 3,45 & 0,39 \\
\hline \multicolumn{8}{|l|}{ Integralidade Serviços Prestados } \\
\hline Alimentos saudáveis ou sono adequado & $0(0,0)$ & $3(0,9)$ & $8(2,4)$ & $53(15,6)$ & $276(81,1)$ & 3,77 & 0,40 \\
\hline $\begin{array}{l}\text { Segurança no lar, como armazenagem segura de } \\
\text { medicamentos }\end{array}$ & $5(1,5)$ & $21(6,2)$ & $32(9,4)$ & $86(25,3)$ & $196(57,6)$ & 3,35 & 0,51 \\
\hline Uso de cintos de segurança & $9(2,6)$ & $130(38,2)$ & $98(28,8)$ & $46(13,5)$ & $57(16,8)$ & 2,10 & 0,44 \\
\hline Abordagem de conflitos familiares & $1(0,3)$ & $16(4,7)$ & $23(6,7)$ & $92(27,0)$ & $208(61,1)$ & 3,45 & 0,39 \\
\hline Aconselhamento sobre exercícios físicos apropriados & $0(0,0)$ & $6(1,8)$ & $5(1,4)$ & $85(25,0)$ & $244(71,8)$ & 3,67 & 0,45 \\
\hline Níveis de colesterol & $0(0,0)$ & $2(0,6)$ & $1(0,3)$ & $49(14,4)$ & $288(84,5)$ & 3,83 & 0,39 \\
\hline Discussão sobre medicações em uso & $0(0,0)$ & $0(0,0)$ & $1(0,3)$ & $31(9,1)$ & $308(90,6)$ & 3,90 & 0,33 \\
\hline $\begin{array}{l}\text { Exposição a substâncias nocivas em casa, no trabalho } \\
\text { ou na vizinhança }\end{array}$ & $5(1,5)$ & $25(7,4)$ & $44(12,9)$ & $114(33,5)$ & $152(44,7)$ & 3,16 & 0,60 \\
\hline $\begin{array}{l}\text { Disponibilidade, armazenamento e segurança de } \\
\text { armas de fogo }\end{array}$ & $10(2,9)$ & $140(41,2)$ & $82(24,1)$ & $59(17,4)$ & $49(14,4)$ & 2,06 & 0,50 \\
\hline Prevenção de queimaduras por água quente & $7(2,1)$ & $63(18,5)$ & $26(7,6)$ & $82(24,1)$ & $162(47,6)$ & 3,02 & 0,62 \\
\hline Prevenção de quedas & $7(2,1)$ & $36(10,5)$ & $21(6,2)$ & $83(24,4)$ & $193(56,8)$ & 3,28 & 0,71 \\
\hline Prevenção de osteoporose em mulheres & $0(0,0)$ & $7(2,1)$ & $6(1,8)$ & $92(27,0)$ & $235(69,1)$ & 3,63 & 0,30 \\
\hline $\begin{array}{l}\text { Cuidado de problemas comuns relativos a } \\
\text { menstruação ou a menopausa }\end{array}$ & $0(0,0)$ & $8(2,4)$ & $4(1,2)$ & $66(19,4)$ & $262(77,1)$ & 3,71 & 0,23 \\
\hline $\begin{array}{l}\text { Maneiras de lidar com os problemas de } \\
\text { comportamento das crianças }\end{array}$ & $3(1,2)$ & $10(4,0)$ & $12(4,8)$ & $67(26,7)$ & $159(63,4)$ & 3,50 & $0,57^{\star \&}$ \\
\hline $\begin{array}{l}\text { Mudanças do crescimento e desenvolvimento da } \\
\text { criança esperadas para cada faixa etária }\end{array}$ & $1(0,4)$ & $6(2,4)$ & $7(2,8)$ & $53(21,1)$ & $184(73,3)$ & 3,66 & $0,58 * 8$ \\
\hline $\begin{array}{l}\text { Segurança (menos } 6 \text { anos): atravessar a rua e uso de } \\
\text { assentos nos carros }\end{array}$ & $5(2,0)$ & $29(11,6)$ & $51(20,3)$ & $81(32,3)$ & $85(33,9)$ & 2,89 & 0,46 *\& \\
\hline $\begin{array}{l}\text { Segurança (entre } 6 \text { e } 12 \text { anos): distância armas, } \\
\text { cintos de segurança e capacetes ciclistas }\end{array}$ & $6(2,4)$ & $35(13,9)$ & $61(24,3)$ & $85(33,9)$ & $64(25,5)$ & 2,72 & $0,43^{* \&}$ \\
\hline
\end{tabular}


Tabela 1. Distribuição das respostas dos itens, escore médio e cargas fatoriais para validade fatorial.

\begin{tabular}{|c|c|c|c|c|c|c|c|}
\hline Itens por atributos & $\begin{array}{l}\text { Não sei/Não } \\
\text { lembro n (\%) }\end{array}$ & $\begin{array}{l}\text { Com certeza } \\
\text { não } \mathrm{n}(\%)\end{array}$ & $\begin{array}{l}\text { Provavelmente } \\
\text { não n (\%) }\end{array}$ & $\begin{array}{l}\text { Provavelmente } \\
\operatorname{sim} n(\%)\end{array}$ & $\begin{array}{l}\text { Com certeza } \\
\operatorname{sim} n(\%)\end{array}$ & $\begin{array}{l}\text { Escore } \\
\text { Médio" }\end{array}$ & $\begin{array}{c}\text { Carga } \\
\text { fatorial }^{*}\end{array}$ \\
\hline $\begin{array}{l}\text { Segurança (acima de } 12 \text { anos): sexo seguro, dizer não } \\
\text { às drogas, não beber e dirigir }\end{array}$ & $1(0,4)$ & $7(2,8)$ & $9(3,6)$ & $65(25,3)$ & $175(68,1)$ & 3,59 & $0,37^{* \&}$ \\
\hline \multicolumn{8}{|l|}{ Orientação Familiar } \\
\hline $\begin{array}{l}\text { Ideias e opiniões do paciente sobre o seu tratamento } \\
\text { e cuidado ou do membro da família }\end{array}$ & $6(1,8)$ & $16(4,7)$ & $23(6,8)$ & $108(31,8)$ & $187(55,0)$ & 3,38 & 0,40 \\
\hline $\begin{array}{l}\text { Pergunta sobre doenças e problemas de saúde nas } \\
\text { famílias dos pacientes }\end{array}$ & $1(0,3)$ & $4(1,2)$ & $5(1,4)$ & $82(24,1)$ & $248(72,9)$ & 3,69 & 0,34 \\
\hline $\begin{array}{l}\text { Disposto para atender membros da família para } \\
\text { discutir problema de saúde ou familiar }\end{array}$ & $0(0,0)$ & $3(0,9)$ & $3(0,9)$ & $71(20,9)$ & $263(77,4)$ & 3,75 & 0,21 \\
\hline $\begin{array}{l}\text { Uso de genogramas e/ou outros instrumentos de } \\
\text { avaliação do funcionamento familiar }\end{array}$ & $5(1,4)$ & $146(42,9)$ & $63(18,5)$ & $61(17,9)$ & $65(19,1)$ & 2,14 & 0,36 \\
\hline $\begin{array}{l}\text { Discussão sobre fatores de risco familiares, ex. } \\
\text { genéticos }\end{array}$ & $1(0,3)$ & $31(9,1)$ & $26(7,6)$ & $98(28,8)$ & $184(54,1)$ & 3,28 & 0,34 \\
\hline $\begin{array}{l}\text { Discussão sobre recursos econômicos da família dos } \\
\text { pacientes }\end{array}$ & $0(0,0)$ & $38(11,1)$ & $30(8,8)$ & $114(33,9)$ & $158(46,4)$ & 3,15 & 0,68 \\
\hline $\begin{array}{l}\text { Discussão sobre fatores de risco sociais, ex. perda de } \\
\text { emprego }\end{array}$ & $0(0,0)$ & $27(7,9)$ & $24(7,1)$ & $122(35,5)$ & $167(49,1)$ & 3,26 & 0,69 \\
\hline $\begin{array}{l}\text { Discussão sobre condições de vida, ex. refrigerador } \\
\text { em condições de funcionamento }\end{array}$ & $3(0,9)$ & $34(10,0)$ & $44(12,9)$ & $121(35,6)$ & $138(40,6)$ & 3,07 & 0,55 \\
\hline $\begin{array}{l}\text { Discussão sobre estado de saúde de outros membros } \\
\text { da família }\end{array}$ & $0(0,0)$ & $13(3,8)$ & $20(5,9)$ & $111(32,6)$ & $196(57,7)$ & 3,44 & 0,60 \\
\hline Discussão sobre as funções parentais & $2(0,6)$ & $26(7,6)$ & $40(11,8)$ & $128(37,6)$ & $144(42,4)$ & 3,15 & 0,66 \\
\hline Avaliação de sinais de abuso infantil & $2(0,6)$ & $39(11,4)$ & $31(9,1)$ & $83(24,4)$ & $185(54,4)$ & 3,22 & 0,43 \\
\hline Avaliação de sinais de crise familiar & $1(0,3)$ & $16(4,7)$ & $19(5,6)$ & $125(36,8)$ & $179(52,6)$ & 3,38 & 0,61 \\
\hline $\begin{array}{l}\text { Avaliação do impacto da saúde do paciente sobre } 0 \\
\text { funcionamento da família }\end{array}$ & $1(0,3)$ & $12(3,5)$ & $22(6,4)$ & $121(35,5)$ & $184(54,1)$ & 3,40 & 0,68 \\
\hline Avaliação do nível de desenvolvimento familiar & $6(1,8)$ & $32(9,4)$ & $45(13,3)$ & $151(44,5)$ & $105(31,0)$ & 2,98 & 0,61 \\
\hline \multicolumn{8}{|l|}{ Orientação Comunitária } \\
\hline Visitas domiciliares & $5(1,4)$ & $7(2,1)$ & $5(1,4)$ & $15(4,4)$ & $308(90,6)$ & 3,84 & 0,17 \\
\hline $\begin{array}{l}\text { Conhecimento adequado dos problemas de saúde da } \\
\text { comunidade que atende }\end{array}$ & $5(1,4)$ & $14(4,1)$ & $29(8,5)$ & $143(42,0)$ & $149(43,8)$ & 3,26 & 0,37 \\
\hline $\begin{array}{l}\text { Opiniões de usuários que possam ajudar no } \\
\text { fornecimento de melhores cuidados de saúde }\end{array}$ & $9(2,6)$ & $16(4,7)$ & $46(13,5)$ & $98(28,8)$ & $171(50,3)$ & 3,26 & 0,44 \\
\hline $\begin{array}{l}\text { Adaptação de serviços ou programas em resposta a } \\
\text { problemas específicos da comunidade }\end{array}$ & $9(2,6)$ & $3(0,9)$ & $19(5,6)$ & $135(39,7)$ & $174(51,1)$ & 3,43 & 0,23 \\
\hline Informações de mortalidade (dados sobre óbitos) & $24(7,1)$ & $26(7,6)$ & $27(7,9)$ & $59(17,4)$ & $204(60,0)$ & 3,33 & 0,51 \\
\hline Dados de doenças de notificação compulsória & $4(1,2)$ & $13(3,8)$ & $11(3,2)$ & $68(20,0)$ & $244(71,8)$ & 3,60 & 0,71 \\
\hline Taxas de imunização da comunidade & $4(1,2)$ & $8(2,4)$ & $7(2,1)$ & $46(13,5)$ & $275(80,5)$ & 3,74 & 0,65 \\
\hline Dados secundários sobre saúde e riscos ocupacionais & $29(8,5)$ & $45(13,2)$ & $72(21,1)$ & $102(30,0)$ & $92(27,0)$ & 2,75 & 0,35 \\
\hline Informações clínicas do próprio serviço & $11(3,2)$ & $9(2,6)$ & $22(6,4)$ & $49(14,4)$ & $249(73,2)$ & 3,60 & 0,56 \\
\hline Pesquisas com os seus pacientes & $24(7,1)$ & $119(35,0)$ & $70(20,5)$ & $62(18,2)$ & $65(19,1)$ & 2,25 & 0,61 \\
\hline Pesquisas na sua comunidade & $30(8,8)$ & $111(32,6)$ & $63(18,5)$ & $61(17,9)$ & $75(22,1)$ & 2,34 & 0,63 \\
\hline $\begin{array}{l}\text { Feedback de organizações comunitárias ou conselhos } \\
\text { gestores de saúde }\end{array}$ & $22(6,4)$ & $46(13,5)$ & $39(11,4)$ & $109(32,0)$ & $124(36,4)$ & 2,95 & 0,62 \\
\hline $\begin{array}{l}\text { Feedback (retorno das informações) da equipe de } \\
\text { saúde }\end{array}$ & $11(3,2)$ & $29(8,5)$ & $24(7,1)$ & $81(23,9)$ & $194(57,2)$ & 3,31 & 0,51 \\
\hline Análise de dados de saúde locais ou estatísticas vitais & $32(9,4)$ & $37(10,9)$ & $40(11,8)$ & $96(28,2)$ & $135(39,7)$ & 3,01 & 0,55 \\
\hline $\begin{array}{l}\text { Avaliações sistemáticas de seus programas e serviços } \\
\text { prestados }\end{array}$ & $18(5,2)$ & $38(11,1)$ & $40(11,8)$ & $103(30,2)$ & $141(41,4)$ & 3,05 & 0,55 \\
\hline
\end{tabular}


Tabela 1. Distribuição das respostas dos itens, escore médio e cargas fatoriais para validade fatorial.

\begin{tabular}{|c|c|c|c|c|c|c|c|}
\hline Itens por atributos & $\begin{array}{l}\text { Não sei/Não } \\
\text { lembro n (\%) }\end{array}$ & $\begin{array}{c}\text { Com certeza } \\
\text { não } \mathrm{n}(\%)\end{array}$ & $\begin{array}{l}\text { Provavelmente } \\
\text { não } \mathrm{n}(\%)\end{array}$ & $\begin{array}{l}\text { Provavelmente } \\
\operatorname{sim} n(\%)\end{array}$ & $\begin{array}{c}\text { Com certeza } \\
\operatorname{sim} n(\%)\end{array}$ & $\begin{array}{l}\text { Escore } \\
\text { Médio }\end{array}$ & $\begin{array}{c}\text { Carga } \\
\text { fatorial }^{*}\end{array}$ \\
\hline Atuação dos Agentes Comunitários de Saúde & $21(6,2)$ & $149(43,7)$ & $8(2,4)$ & $33(9,7)$ & $129(37,9)$ & 2,45 & 0,45 \\
\hline $\begin{array}{l}\text { Presença de usuários no Conselho Gestor ou Conselho } \\
\text { Distrital de Saúde }\end{array}$ & $41(12,0)$ & $34(10,0)$ & $23(6,8)$ & $56(16,4)$ & $186(54,7)$ & 3,22 & 0,45 \\
\hline $\begin{array}{l}\text { Rede com agências estatais e locais envolvidas com } \\
\text { grupos culturalmente diversos }\end{array}$ & $48(14,1)$ & $65(19,1)$ & $66(19,4)$ & $74(21,8)$ & $87(25,5)$ & 2,61 & 0,38 \\
\hline Vínculos com serviços/organizações religiosas & $39(11,4)$ & $115(33,8)$ & $80(23,5)$ & $59(17,4)$ & $47(13,8)$ & 2,17 & 0,34 \\
\hline $\begin{array}{l}\text { Envolvimento com associações de moradores/ } \\
\text { lideranças comunitários }\end{array}$ & $24(7,1)$ & $12(3,5)$ & $29(8,5)$ & $91(26,8)$ & $184(54,1)$ & 3,35 & 0,45 \\
\hline $\begin{array}{l}\text { Agentes comunitários ou membros do conselho } \\
\text { gestor/conselho distrital de saúde }\end{array}$ & $30(8,8)$ & $35(10,2)$ & $16(4,7)$ & $70(20,5)$ & $189(55,5)$ & 3,26 & 0,54 \\
\hline
\end{tabular}

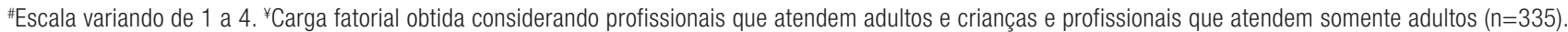
*Itens específicos sobre 0 cuidado com as crianças. ${ }^{8}$ Carga fatorial obtida na análise fatorial adicional (não mostrada), considerando somente profissionais que atendem adultos e crianças $(n=251)$.

Tabela 2. Distribuição de itens dos atributos (ou componentes) da APS nos fatores obtidos pela análise fatorial.

\begin{tabular}{cclc}
\hline Fator & Número de itens & \multicolumn{1}{c}{ Atributo (ou componente) } & Variação explicada* $(\%)$ \\
\hline 1 & 14 & Orientação Familiar & 35,11 \\
2 & 17 & Orientação Comunitária & 8,19 \\
3 & 13 & Integralidade Serviços Prestados & 6,10 \\
4 & 4 & Orientação Comunitária & 5,49 \\
5 & 4 & Acesso de Primeiro Contato & 5,02 \\
& 7 & Longitudinalidade & \\
6 & 7 & Coordenação do cuidado & 4,26 \\
7 & 8 & Coordenação sistema de informação & \\
& 16 & Integralidade Serviços Disponíveis & 3,89 \\
9 & 5 & Acesso de Primeiro Contato & 3,30 \\
& 6 & Logitudinalidade & 3,23 \\
\hline
\end{tabular}

${ }^{*}$ Obtida considerando profissionais que atendem adultos e crianças e profissionais que atendem somente adultos $(n=335)$.

A avaliação da consistência interna pelo alfa de Cronbach foi adequada para Longitudinalidade, Integralidade de Serviços Disponíveis, Integralidade de Serviços, Orientação Familiar e Orientação Comunitária. Para Coordenação Sistema de Informação, o coeficiente alfa de Cronbach foi igual a 0,56, considerado moderado, e para a Coordenação do Cuidado e o Acesso de Primeiro Contato, o alfa foi insuficiente, 0,48 e 0,28, respectivamente (Tabela 3).

Tabela 3. Medidas de fidedignidade e discriminação para os atributos da APS.

\begin{tabular}{|c|c|c|c|c|c|c|c|}
\hline \multirow[b]{2}{*}{ Atributos da Atenção Primária à Saúde } & \multirow[b]{2}{*}{$\mathrm{n}$} & \multirow[b]{2}{*}{$\begin{array}{l}\text { Número de } \\
\text { itens }\end{array}$} & \multirow[b]{2}{*}{$\begin{array}{l}\text { Alfa de } \\
\text { Cronbach }\end{array}$} & \multirow[b]{2}{*}{$\begin{array}{l}\text { Correlação item- } \\
\text { total (Mín - Máx) }\end{array}$} & \multicolumn{2}{|c|}{ Medidas Teste-Reteste } & \multirow[b]{2}{*}{$\mathrm{REE}^{*}$} \\
\hline & & & & & $\begin{array}{l}\text { Comparação de médias } \\
\text { ou das distribuições }{ }^{\#}\end{array}$ & $\begin{array}{c}\text { Concordância Bland } \\
\text { Altman }^{\&}\end{array}$ & \\
\hline Acesso de Primeiro Contato & 340 & 9 & 0,28 & $-0,06-0,22$ & 0,892 & 0,781 & $74 \%$ \\
\hline Longitudinalidade & 339 & 13 & 0,72 & $0,14-0,51$ & 0,900 & 0,992 & $92 \%$ \\
\hline Coordenaç̧ão do Cuidado & 340 & 7 & 0,48 & $0,08-0,39$ & 0,251 & 0,113 & $79 \%$ \\
\hline Coordenação Sistema de Informação & 340 & 8 & 0,56 & $0,07-0,43$ & 0,104 & 0,070 & $81 \%$ \\
\hline Integralidade - Serviços Disponíveis & 339 & 22 & 0,78 & $0,07-0,59$ & 0,879 & 0,788 & $89 \%$ \\
\hline Integralidade - Serviços Prestados ${ }^{Q}$ & 340 & 13 & 0,84 & - & - & - & $99 \%$ \\
\hline Integralidade - Serviços Prestados* & 251 & 18 & 0,88 & $0,31-0,69$ & 0,061 & 0,068 & $99 \%$ \\
\hline Orientação Familiar & 339 & 14 & 0,89 & $0,40-0,71$ & 0,003 & 0,001 & $100 \%$ \\
\hline Orientação Comunitária & 339 & 21 & 0,90 & $0,27-0,72$ & 0,658 & 0,702 & $100 \%$ \\
\hline
\end{tabular}

\#Valor-p associado ao teste t ou Wilcoxon para amostras pareadas. ${ }^{\circledR}$ Valor-p associado ao teste t para amostras pareadas. ${ }^{*}$ Razão Êxito da Escala. ${ }^{0}$ Foram considerados profissionais que atendem adultos e crianças e profissionais que atendem somente adultos. *Foram considerados somente profissionais que atendem adultos e crianças. 
A correlação item-total mostrou resultados adequados (mínimo-máximo) para Orientação Familiar e Integralidade de Serviços Prestados. Para Orientaçáo Comunitária, somente um item apresentou correlaçáo item-total não adequada $(0,27-0,72)$. Para Longitudinalidade $(0,14-0,51)$, Coordenação do Cuidado (0,08-0,39), Coordenação Sistema de Informação $(0,07-0,43)$ e Integralidade Serviços Disponíveis (0,07-0,59), foram obtidas tanto correlaçôes não adequadas quanto correlaçóes adequadas. Por outro lado, o Acesso de Primeiro Contato $(-0,06-0,22)$ apresentou correlaçóes item-total náo adequadas para todos os itens que constituem o atributo (Tabela 3).

Os atributos Orientação Familiar e Orientação Comunitária apresentaram REE igual a 100\%. Em contrapartida, os menores percentuais para essa medida foram obtidos no Acesso de Primeiro Contato (REE=74\%) e na Coordenação do Cuidado (REE=79\%). Na fidedignidade relacionada à estabilidade ao longo do tempo, tanto no método de comparação das distribuiçôes quanto no método que observa a concordância entre os diferentes tempos, a estabilidade da escala foi verificada, exceto para o atributo Orientação Familiar $(\mathrm{p}<0,05)$ (Tabela 3).

\section{Discussão}

Neste estudo, foram realizadas tradução, adaptação e avaliação da validade e da fidedignidade do PCATool-Brasil versão profissionais de saúde, evidenciando que o instrumento é válido e fidedigno para a mensuração da qualidade dos serviços de saúde de APS.

$\mathrm{Na}$ validade fatorial, os seis atributos da APS foram captados pelos primeiros 9 fatores na análise fatorial, identificando a natureza multidimensional do instrumento e conceitual do fenômeno, também presente na versão original do instrumento. $\mathrm{O}$ atributo Longitudinalidade foi captado em 2 fatores, os quais também representam os atributos Acesso de Primeiro Contato e Coordenaçã́o. Isso sugere uma forte relação entre o Acesso de um serviço de saúde e a Longitudinalidade que, por sua vez, também está fortemente relacionada à Coordenação do serviço (Tabela 2).

Além disso, os itens que representam a confiança usuário-profissional (processo da Longitudinalidade) mostraram-se relacionados ao Acesso de Primeiro Contato, enquanto que os itens sobre a continuidade da atençáo (estrutura da Longitudinalidade) foram relacionados à Coordenação do serviço, uma vez que o atributo Longitudinalidade é constituído pelos itens que caracterizam a relaçáo de confiança do usuário (paciente) com um profissional, em particular, e também a continuidade da atenção ao cuidado ${ }^{4}$ (Tabela 2). Essa inter-relação de itens que representam distintos atributos em um mesmo fator ressalta a adequação do instrumento ao conceito de APS, no qual os atributos podem ser operacionalmente segmentados, mas se apresentam, na realidade dos serviços, completamente interligados.

Os resultados para a fidedignidade sugerem consistência para Longitudinalidade, Integralidade, Orientação Familiar e Orientação Comunitária. Por outro lado, a consistência interna foi moderada para a Coordenação e insuficiente para o Acesso de Primeiro Contato, haja vista que as estimativas do alfa de Cronbach foram relativamente baixas. Na REE, os atributos Longitudinalidade, Integralidade, Orientação Familiar e Orientaçáo Comunitária apresentaram os resultados mais elevados, sugerindo ter melhor discriminaçáo. A estabilidade no tempo foi verificada para todos os atributos, exceto a Orientação Familiar. Neste caso, a alteração na estabilidade temporal pode ter sido ocasionada devido a novas experiências dos profissionais com os usuários no serviço de saúde. (Tabela 3).

Nos atributos Acesso de Primeiro Contato e Coordenação, houve predomínio de itens com carga fatorial baixa e medidas de consistência interna insuficiente e moderada, sugerindo a presença de itens com potencial para exclusão. No entanto, a maioria desses itens refere-se essencialmente às características de organização dos serviços de saúde (dias e horários de funcionamento das unidades de saúde) e a transição de usuários entre os níveis primário, secundário e terciário. Essas características, em Porto alegre, são estabelecidas pela administração municipal nos serviços públicos de saúde e, consequentemente, apresentam-se de maneira uniforme nos quatro serviços de APS. As unidades de saúde não funcionam durante a noite, nem nos finais de semana, e a marcação de consultas de referência para outro nível de atenção é realizada por uma central de marcação de consultas, independentemente do serviço ao qual o usuário é afiliado. Consoante a isso, os itens como atendimento nos finais de semana, após as oito horas da noite, ou no mesmo dia, quando o serviço está fechado, e a disponibilidade de telefone para contato foram acentuadamente avaliados de forma negativa. Evidências similares foram encontradas por Mead et al. ${ }^{8}$ bem como por Haggerty et al. ${ }^{17}$, principalmente no que diz respeito à disponibilidade de um número de telefone para contato.

Nesse sentido, a organização dos serviços em Porto Alegre contribui para a alta concentração de respostas em somente uma das opçóes da escala, apontando a total presença ou ausência da característica apresentada no item e ilustrando a sua 
baixa variabilidade (Tabela 1). Segundo Streiner ${ }^{28}$, itens desse tipo tendem a ser pouco discriminantes e pouco contribuem para os resultados da avaliação de escalas. Entretanto, é necessário considerar as particularidades na organização dos serviços, uma vez que a baixa discriminação de itens observada neste estudo não necessariamente significa que sejam inadequados, pois em contextos com diferentes formas de organização dos serviços, podem ser úteis para discriminar a orientação à APS. Também é importante lembrar que características exclusivas dos serviços de saúde, como o fornecimento de informação escrita para levar ao especialista, muitas vezes fundamentais na operacionalização do processo de cuidado, devem estar presentes em processos avaliativos.

Embora os resultados tenham mostrado que alguns itens não possuem todas as propriedades psicométricas desejáveis, optou-se por mantê-los no instrumento devido à importância conceitual para a definição de APS proposta por Starfield ${ }^{4}$, principalmente no caso dos atributos essenciais Acesso de Primeiro Contato e Coordenação. Essas fragilidades podem ser aceitas também por tratar-se de um instrumento que almeja medir numerosas e diversas características de diferentes sistemas de saúde, mantendo a equivalência com instrumentos de avaliação semelhantes aos de outros países.

No Brasil está disponível outra adaptação do PCATool para profissionais de saúde. Almeida e Macinko ${ }^{15}$ validaram essa versão utilizando uma amostra de 33 profissionais, com modificaçôes em relação ao formato original do instrumento. Os atributos essenciais e derivados foram mantidos, à exceção de Competência Cultural. Em contrapartida, foi proposta a inclusão do atributo que se refere à formação profissional. A escala de respostas é a do tipo Likert (de 1=nunca a $6=$ sempre) e a opção 88 (não sabe), sendo, portanto, diferente da escala original. A fidedignidade foi avaliada exclusivamente pelo coeficiente alfa de Cronbach para cada item e também para cada atributo. A validade fatorial foi verificada pela unidimensionalidade, não contemplando a estrutura conceitual (multidimensional) que possibilita a avaliação individual dos atributos da APS ${ }^{15}$. Por conseguinte, o processo de validade e fidedignidade é diferente da abordagem e metodologia utilizada neste estudo, assim como são distintas as adaptaçóes. Outros estudos de validação do PCATool para profissionais de saúde que permitissem contextualizar estes resultados não são de nosso conhecimento.

A origem dos dados de um único município sugere uma limitação na generalização dos resultados, refletindo, potencialmente, validade e fidedignidade das características dos serviços públicos de APS em Porto Alegre. Também parece importante observar o grande número de itens do instrumento e a frequência de respostas na categoria 'não sei/não lembro'. Os maiores percentuais desta categoria foram observados para Orientação Comunitária, sendo a taxa mais elevada de 14,1\%, no item "Rede com agências estatais e locais envolvidas com grupos culturalmente diversos" (Tabela 1). Frequências muito elevadas para a opção 'não sei/não lembro' podem influenciar a qualidade das respostas ao longo do questionário.

A utilização do PCATool exige a avaliação prévia de equivalência com a versão original e a descrição das suas propriedades psicométricas. Do contrário, versôes muito diferentes ocasionam dificuldades para comparaçôes de resultados entre estudos em âmbito nacional ou internacional.

\section{Conclusões}

Mesmo considerando as diferenças regionais e o grande número de itens, o PCATool-Brasil versão profissionais captou os principais atributos da APS e apresentou, de modo geral, medidas de fidedignidade aceitáveis, diante da realidade na qual o instrumento foi aplicado. Assim, o PCATool-Brasil pode ser considerado um instrumento válido e fidedigno para avaliar a presença e extensão dos atributos da APS na experiência dos profissionais de saúde. Por conseguinte, é um importante instrumento para avaliar serviços de saúde, subsidiando gestores sobre a presença e extensão dos atributos da APS.

Em estudos comparativos pode-se utilizar a versão em espelho dos usuários, com número menor de itens, conforme sugerido pela autora do instrumento original. Para minuciosa avaliação local da presença e extensão dos atributos da APS, sugere-se a utilização do PCATool-Brasil versão profissionais de saúde cuja validade e fidedignidade foram averiguadas neste estudo. Embora seja um instrumento um pouco extenso, permite identificar especificamente possíveis indicadores de baixa qualidade que necessitam de açôes e/ou monitoramento. 


\section{Agradecimentos}

Este artigo faz parte de um projeto financiado pela Fundação de Amparo à Pesquisa do Estado do Rio Grande do Sul (FAPERGS), por meio do edital 008/2004, para Projetos de Pesquisa e Desenvolvimento Prioritários para o Sistema Único de Saúde (processo 04/1507.3), pelo Conselho Nacional de Desenvolvimento Científico e Tecnológico (CNPq), por meio do edital 49/2005 (processo 402466/2005-5), e pela Coordenação de Aperfeiçoamento de Pessoal de Nível Superior do Ministério da Educação (CAPES), por meio de bolsa PRODOC, 2005.

\section{Referências}

1. Starfield B, Shi L, Macinko J. Contribution of Primary Care to Health Systems and Health. Milbank Q. 2005; 83: 457-502. PMid:16202000 PMCid:PMC2690145. http://dx.doi.org/10.1111/j.1468-0009.2005.00409.x

2. Shi L, Macinko J, Starfield B, Politzer R, Xu J. Primary care, race, and mortality in US states. Soc Sci Med. 2005; 61: 65-75. PMid:15847962. http:// dx.doi.org/10.1016/j.socscimed.2004.11.056

3. Campbell S, Roland M, Buetow S. Defining quality of care. Soc Sci Med. 2000; 51: 1611-25.

4. Starfield B. Atenção primária: equilíbrio entre necessidades de saúde, serviços e tecnologia. Brasília: Organização das Nações Unidas para a Educação; Ministério da Saúde; 2002

5. Haggerty J, Burge F, Levesque JF, Gass D, Pineault R, Beaulieu MD, et al. Operational definitions of attributes of primary health care: consensus among Canadian experts. Ann Fam Med. 2007; 5: 336-44. PMid:17664500 PMCid:PMC1934980. http://dx.doi.org/10.1370/afm.682

6. Hussey PS, De Vries H, Romley J, Wang MC, Chen SS, Shekelle PG, et al. A systematic review of health care efficiency measures. Health Serv Res. 2009; 44: 784-805. PMid:19187184 PMCid:PMC2699907. http://dx.doi.org/10.1111/j.1475-6773.2008.00942.x

7. Ohman-Strickland PA, John OA, Nutting PA, Dickinson WP, Scott-Cawiezell J, Hahn K, et al. Measuring organizational attributes of primary care practices: development of a new instrument. Health Serv Res. 2007; 42: 1257-73. PMid:17489913 PMCid:PMC1955254. http://dx.doi.org/10.1111/ j.1475-6773.2006.00644.x

8. Mead N, Bower P, Roland M. The General Practice Assessment Questionnaire (GPAQ)-development and psychometric characteristics. BMC Fam Pract. 2008; 9: 13. PMid:18289385 PMCid:PMC2277420. http://dx.doi.org/10.1186/1471-2296-9-13

9. Shi L, Starfield B, Xu J. Validating the Adult Primary Care Assessment Tool. J Fam Pract. 2001; 50: 161W-175W.

10. Malouin R, Starfield B, Sepulveda M. Evaluating the tools used to assess the medical home. Manag Care. 2009; 18(6):44-8. PMid:19569570.

11. Cassady C, Starfield B, Hurtado M, Berk R, Nanda J, Friedenberg L. Measuring consumer experiences with primary care. Pediatrics. 2000; 105:998-1003.

12. Starfield B, Shi L. Manual for the Primary Care Assessment Tools. Baltimore: Johns Hopkins University; 2002.

13. Harzheim E, Starfield B, Rajmil L, Álvarez-Dardet C, Stein A. Internal consistency and reliability of Primary Care Assessment Tool (PCATool-Brasil) for child health services. Cad Saude Pública. 2006; 22(8): 1649-59. PMid:16832536. http://dx.doi.org/10.1590/S0102-311X2006000800013

14. Harzheim E, Gonçalves MR, Oliveira MMC, Trindade TG, Agostinho MR, Hauser L. Manual do instrumento de avaliação da atenção primária à saúde: primary care assessment tool PCATool - Brasil. Brasília: Ministério da Saúde; 2010. [acesso em 30 mar 2013]. Disponível em: http://189.28.128.100/ dab/docs/publicacoes/geral/manual_instrumento_avaliacao.pdf

15. Almeida C, Macinko J. Validação de uma metodologia de avaliação rápida das características organizacionais e do desempenho dos serviços de atenção básica do Sistema Único de Saúde (SUS) em nível local. Brasília: Organização Pan-Americana da Saúde; 2006.

16. Pasarin MI, Berra S, Rajmil L, Solans M, Borrell C, Starfield B. [An instrument to evaluate primary health care from the population perspective] (in Spanish). Aten Primaria. 2007; 39: 395-401. PMid:17692225. http://dx.doi.org/10.1157/13108612

17. Haggerty JL, Pineault R, Beaulieu MD, Brunelle Y, Gauthier J, Goulet F, et al. Practice features associated with patient-reported accessibility, continuity, and coordination of primary health care. Ann Fam Med. 2008; 6: 116-23. PMid:18332403 PMCid:PMC2267415. http://dx.doi.org/10.1370/afm.802

18. Lee JH, Choi YJ, Sung NJ, Kim S, Chung SH, Kim J, et al. Development of the Korean primary care assessment tool--measuring user experience: tests of data quality and measurement performance. Int J Qual Health Care. 2009 Apr; 21(2): 103-11. PMid:19286829. http://dx.doi.org/10.1093/ intqhc/mzp007

19. Wong SY, Kung K, Griffiths SM, Carthy T, Wong MC, Lo SV, et al. Comparison of primary care experiences among adults in general outpatient clinics and private general practice clinics in Hong Kong. BMC Public Health. 2010; 10: 397. PMid:20602806 PMCid:PMC2908092. http://dx.doi. org/10.1186/1471-2458-10-397

20. Berra S, Audisio Y, Mántaras J, Nicora V, Mamondi V, Starfield B. Adaptación cultural y al sistema de salud argentino del conjunto de instrumentos para la evaluación de la atención primaria en salud. Rev. Argent. Salud Publica 2011; 2(8): 6-14.

21. Puerto Rico Health Services Research Institute. Development of a Spanish Version of the Primary Care Assessment Tool (PCAT). [acesso em 31 mar 2012]. Disponível em: prhsri.rcm.upr.edu/recentpro.html 
22. Pizanelli M, Ponzo J, Buglioli M, Toledo A, Casinelli M, Gómez A. Validación de Primary Care Assessment Tool (PCAT) en Uruguay. Rev Med Urug. 2011; 27(3): 187-189.

23. Jatrana S, Crampton P, Richardson K. Continuity of care with general practitioners in New Zealand: results from SoFIE-Primary Care. N Z Med J. 2011 Feb 11; 124(1329): 16-25. PMid:21475356.

24. Tsai J, Shi L, Yu WL, Hung LM, Lebrun LA. Physician specialty and the quality of medical care experiences in the context of the Taiwan national health insurance system. J Am Board Fam Med. 2010; 23(3): 402-12. PMid:20453187. http://dx.doi.org/10.3122/jabfm.2010.03.090222

25. Berra S, Rocha KB, Sanz MR, Pasarín MI, Rajmil L, Borrel C, et al. Properties of a short questionnaire for assessing Primary Care experiences for children in a population survey. BMC Public Health. 2011; 11: 285.

26. Macinko J, Starfield B, Shi L. The contribution of primary care systems to health outcomes within Organization for Economic Cooperation and Development (OECD) countries, 1970-1998. Health Serv Res. 2003; 38: 831-65. PMid:12822915 PMCid:PMC1360919. http://dx.doi. org/10.1111/1475-6773.00149

27. Harzheim E, Duncan B, Stein A, Cunha C, Goncalves M, Trindade T, et al. Quality and effectiveness of different approaches to primary care delivery in Brazil. BMC Health Serv Res. 2006; 5(6): 156. PMid:17147819 PMCid:PMC1790713. http://dx.doi.org/10.1186/1472-6963-6-156

28. Streiner DL, Norman GR. Health Measurement Scales: A Practical Guide to Their Development and Use. 4th ed. Oxford University Press; 2008. 